\title{
Bäcklund Transformation, Lax Pair and Solitons of the (2+1)- dimensional Davey-Stewartson-like Equations with Variable Coefficients for the Electrostatic Wave Packets
}

Hui-Ping Zhou, Bo Tian, Hui-Xia Mo, Min Li, Pan Wang

To cite this article: Hui-Ping Zhou, Bo Tian, Hui-Xia Mo, Min Li, Pan Wang (2013) Bäcklund Transformation, Lax Pair and Solitons of the (2+1)-dimensional DaveyStewartson-like Equations with Variable Coefficients for the Electrostatic Wave Packets, Journal of Nonlinear Mathematical Physics 20:1, 94-105, DOI:

https://doi.org/10.1080/14029251.2013.792475

To link to this article: https://doi.org/10.1080/14029251.2013.792475

Published online: 04 January 2021 


\title{
Bäcklund Transformation, Lax Pair and Solitons of the (2+1)-dimensional Davey-Stewartson-like Equations with Variable Coefficients for the Electrostatic Wave Packets
}

\author{
Hui-Ping Zhou ${ }^{1}$, Bo Tian ${ }^{2}$, Hui-Xia Mo ${ }^{1 *}, \mathrm{Min} \mathrm{Li}^{2}$, Pan Wang ${ }^{2}$ \\ ${ }^{1}$ School of Science, Beijing University of Posts and Telecommunications, P.O. Box 122 \\ Beijing, 100876, the People's Republic of China \\ ahuiping@126.com,huixmo@bupt.edu.cn \\ ${ }^{2}$ Ministry of Education, Key Laboratory of Information Photonics and Optical Communications (BUPT), P.O. Box 128 \\ Beijing, 100876, the People's Republic of China \\ tian.bupt@yahoo.com.cn, li.xiao.min85@gmail.com,wangpanpan19851105@yahoo.com.cn \\ Received 14 October 2012 \\ Accepted 13 January 2013
}

\begin{abstract}
The (2+1)-dimensional Davey-Stewartson-like equations with variable coefficients have the applications in the ultra-relativistic degenerate dense plasmas and Bose-Einstein condensates. Via the Bell polynomials and symbolic computation, the bilinear form, Bäcklund transformation and Lax pair for such equations are obtained. Based on the Hirota method, we construct the soliton solutions, analyze the elastic collisions with the constant and variable coefficients, and observe that solitons no longer keep rectilinear propagation and display different shapes because of the variable coefficients. Besides, localized excitations are derived through the variable separation.
\end{abstract}

Keywords: Davey-Stewartson-like equations; Bell polynomials; Symbolic computation; Hirota method; Bäcklund transformation; Lax pair; Solitons; Localized excitations.

PACS numbers: 05.45.Yv, 52.35.Mw, 52.35.Sb

\section{Introduction}

Able to describe a (2+1)-dimensional wave packet on the surface of a liquid of finite depth, DaveyStewartson (DS) equations are written as [5]

$$
\begin{aligned}
& i A_{T}+\sigma^{2}\left(A_{X X}+\sigma^{2} A_{Y Y}\right)+\alpha|A|^{2} A-A B=0, \\
& B_{X X}-\sigma^{2} B_{Y Y}=2 \alpha|A|_{X X}^{2}, \quad \sigma^{2}= \pm 1, \quad \alpha= \pm 1,
\end{aligned}
$$

where the subscripts denote the partial derivatives, $X$ is the scaled horizontal coordinate, $Y$ denotes the scaled space coordinate perpendicular to $X, T$ is the scaled time, the complex function $A$ is the amplitude of a surface wave packet, while the real function $B$ is the velocity potential of the mean flow interacting with the surface wave. The case $\sigma=1$ is called the DSI equations, while $\sigma=i$, the DSII [27]. The parameter $\alpha=1$ characterizes the focusing case, while $\alpha=-1$, the defocusing [27].

Studies have been made on the solutions and integrability of Eqs. (1.1) $[1,4,5,8,15,18,22$ $24,27]$. Eqs. (1.1) can provide certain (2+1)-dimensional nonlinear localized excitations [18]. A class of the (2+1)-dimensional nonlinear dispersive equations can be reduced to Eqs. (1.1) through

${ }^{*}$ Corresponding author. 
the asymptotic considerations [6]. As the extension of the (1+1)-dimensional nonlinear Schrödinger equation, Eqs. (1.1) are shown to be integrable in some senses, for example, the inverse-scatteringtransformation (IST) integrable [1, 4, 22] and Painlevé integrable [8]. In Ref. [15], the bilinear form and dromion solutions have been constructed via the Hirota method. The Bäcklund transformation (BT) of Eq. (1.1) have been obtained through the Zakharov and Shabat method [4, 23]. By means of the iteration transformations, the Bäcklund-Darboux transformations of Eq. (1.1) have been given [24, 27]. In Refs. [7, 26], the soliton solutions have been shown to be representable in terms of the Wronskian determinants. Localized excitations via a variable separation approach have been derived [33]. Moveover, time evolution of solutions has been investigated via the numerical analysis and simulations $[3,19,27]$.

Constant-coefficient DS-like equations have been found in optics [13, 14, 36], plasmas [29-31], quantum physics [10] and Bose-Einstein condensates [18, 32]. For example, the constant-coefficient DS-like equations which describe the slow modulation of $(2+1)$-dimensional electrostatic wave packets in the ultra-relativistic degenerate dense plasmas are given as $[29,30]$

$$
\begin{aligned}
& i \omega_{\tau}+\chi_{1} \omega_{\xi \xi}+\chi_{2} \omega_{\eta \eta}+\chi_{3}|\omega|^{2} \omega+\chi_{4} \omega v=0, \\
& \chi_{5} v_{\xi \xi}+v_{\eta \eta}=\chi_{6}|\omega|_{\xi \xi}^{2},
\end{aligned}
$$

where $\xi$ is a normalized horizontal propagation direction of the electrostatic wave packets, $\eta$ is normalized space propagation direction perpendicular to $\xi, \tau$ is the normalized time, $\omega$ is the complex amplitude of electrostatic wave packets, the real function $v$ is the a static field generated due to the mean motion in plasmas, $\chi_{i}$ 's $(i=1, \cdots, 6)$ are the constants only in terms of the wave number and regime's density, $\chi_{1}$ and $\chi_{2}$ appear due to the wave group dispersion and the evolution of the electrostatic wave packets, cubic nolinear coefficient $\chi_{3}$ denotes the effect of the carrier wave self-interaction due to the zeroth harmonic modes, nonlocal quadratic coefficient $\chi_{4}$ represents the coupling strength between the dynamical field associated with the first harmonic and a static field generated due to the mean motion in the plasmas, while $\chi_{5}$ and $\chi_{6}$ are decided respectively by the first harmonic field and the zeroth harmonic static field of the plasmas. Eqs. (1.2) may give rise to some unstable solutions other than the localization [29].

Based on Eqs. (1.1-1.2), we will consider the variable-coefficient DS-like equations as

$$
\begin{aligned}
& i u_{t}+P_{1}(t) u_{x x}+P_{2}(t) u_{y y}-Q_{1}(t)|u|^{2} u+Q_{2}(t) u \phi=0, \\
& R \phi_{x x}-\phi_{y y}=S|u|_{x x}^{2},
\end{aligned}
$$

where the waves propagate in the two surface directions $x$ and $y$ with the scaled time $t$, the real functions $P_{1}(t)$ and $P_{2}(t)$ represent the wave group dispersion, $Q_{1}(t)$ is the cubic nolinear coefficient and $Q_{2}(t)$ stands for the nonlocal quadratic nonlinearity, $R$ and $S$ are constants, $u=u(x, y, t)$ is the complex wave envelope and the real function $\phi=\phi(x, y, t)$ can be regarded as a forcing term. Special cases of Eqs. (1.3) include the following:

- When $P_{1}(t)=1, P_{2}(t)=1, Q_{1}(t)=-\alpha, Q_{2}(t)=-1, R=1$ and $S=2 \alpha$, reducible to Eqs. (1.1) with $\sigma=1$;

- When $P_{1}(t)=-1, P_{2}(t)=1, Q_{1}(t)=-\alpha, Q_{2}(t)=-1, R=-1$ and $S=-2 \alpha$, reducible to Eqs. (1.1) with $\sigma=i$;

- When $P_{1}(t)=\chi_{1}, P_{2}(t)=\chi_{2}, Q_{1}(t)=-\chi_{3}, Q_{2}(t)=\chi_{4}, R=-\chi_{5}$ and $S=-\chi_{6}$, reducible to Eqs. (1.2); 
- When $2 P_{1}(t)=2 P_{2}(t)=2 Q_{1}(t)=Q_{2}(t)=\beta(t)$ and $R=S=1$, where $\beta(t)$ is real function with time variable $t$, reducible to the equations considered in Ref. [35], whose excitation solutions are obtained via the variable separation approach [25].

Our paper will be organized as follows: In Sec. II, Eqs. (1.3) will be bilinearized via the Bell polynomials and symbolic computation. In Sec. III, BT and Lax pair for Eqs. (1.3) will be obtained from the Bilinear form. In Sec. IV, based on the Hirota method, the soliton solutions of Eqs. (1.3) will be constructed, and the elastic collisions for the soliton solutions with the constant and variable coefficients will be analyzed. Besides, the localized excitations of the Eqs. (1.3) will be derived through the variable separation. Finally, conclusions will be given in Sec. V.

\section{Bilinear Form for Eqs. (1.3)}

Bell polynomials, sometimes, provide a relatively convenient way to obtain the bilinear forms, BTs and Lax pairs for nonlinear partial differential equations $[2,12,20]$. Assuming that $h$ is a $C^{\infty}(z)$ function of $z$, and $h_{k}=\partial_{z}^{k} h(k=1,2, \ldots)$, the Bell polynomials $[2,12,20,21]$ are defined as follows:

$$
Y_{n z}(h) \equiv Y_{n}\left(h_{1}, h_{2}, \ldots, h_{n}\right)=e^{-h} \partial_{z}^{n} e^{h}, \quad n=1,2, \ldots
$$

We can similarly get the two-dimensional generalization of the Bell polynomials:

$$
Y_{n z_{1}, m z_{2}}(Z) \equiv Y_{n, m}\left(Z_{i, j}\right)=e^{-Z} \partial_{z_{1}}^{n} \partial_{z_{2}}^{m} e^{Z}, \quad i=1,2, \ldots, n, \quad j=1,2, \ldots, m,
$$

where $Z$ is a $C^{\infty}$ function of $z_{1}, z_{2}$, and $Z_{i, j}=\partial_{z_{1}}^{i} \partial_{z_{2}}^{j} Z(i=1,2, \ldots, n, j=1,2, \ldots, m)$ with $n$ and $m$ as the non-negative integers.

Ref. [12] has shown that the $D$ operator and Bell polynomial have the relation as a result of their actions on a pair of the exponentials $F=\exp \left[f\left(z_{1}, \ldots, z_{l}\right)\right]$ and $G=\exp \left[g\left(z_{1}, \ldots, z_{l}\right)\right]$ :

$$
\left.(F \cdot G)^{-1} D_{x}^{n} F \cdot G \equiv Y_{n}\left(h_{1}, h_{2}, \ldots, h_{n}\right)\right|_{h_{k}=\left\{\begin{array}{l}
w_{k x}, k=\text { even } \\
v_{k x}, k=\text { odd }
\end{array}\right.} \equiv \mathscr{Y}_{n x}(w, v),
$$

where $w=\ln F+\ln G=\ln (F G), v=\ln F-\ln G=\ln (F / G)$, and the bilinear differential operators $D[17]$ is defined by

$$
D_{\rho}^{m} D_{\varsigma}^{n} \Phi(\rho, \varsigma) \cdot \Psi(\rho, \varsigma)=\left.\left(\frac{\partial}{\partial \rho}-\frac{\partial}{\partial \rho^{\prime}}\right)^{m}\left(\frac{\partial}{\partial \varsigma}-\frac{\partial}{\partial \varsigma^{\prime}}\right)^{n} \Phi(\rho, \varsigma) \Psi\left(\rho^{\prime}, \varsigma^{\prime}\right)\right|_{\rho^{\prime}=\rho, \varsigma^{\prime}=\varsigma} .
$$

According to the formulas above, we can bilinearize Eqs. (1.3) into the binary-Bell-polynomial form.

By setting

$$
u=F / G, \quad \phi=2 C(\ln G)_{x x}, \quad(C=\text { const })
$$

with the assumptions

$$
v=\ln (F / G), \quad w=\ln (F G), \quad \varphi=w-v=2 \ln G,
$$


we can decouple Eqs. (1.3) into the following binary-Bell-polynomial form (for simplification, we assume that $\left.Q_{1}(t) / P_{2}(t)=K\right)$ :

$$
\begin{aligned}
& i \mathscr{Y}_{t}(v)+P_{1}(t) \mathscr{Y}_{2 x}(v, w)+P_{2}(t) \mathscr{Y}_{2 y}(v, w)=0 \\
& R \mathscr{P}_{2 x}(\varphi)-\mathscr{P}_{2 y}(\varphi)=K e^{v+v^{*}}
\end{aligned}
$$

while the corresponding bilinear form is

$$
\begin{aligned}
& {\left[i D_{t}+P_{1}(t) D_{x}^{2}+P_{2}(t) D_{y}^{2}\right] F \cdot G=0,} \\
& \left(R D_{x}^{2}-D_{y}^{2}\right) G \cdot G=K F F^{*},
\end{aligned}
$$

with the coefficient constraints

$$
C=S / K, \quad K P_{1}(t)=S Q_{2}(t)-R Q_{1}(t) .
$$

Especially, Eqs. (2.8) explain the choice of the equations' coeffcients in Ref. [35]. From Eqs. (2.7), we can not only obtain the BT and Lax pair, but also construct the $N$-soliton solutions.

\section{BT and Lax Pair}

\section{1. $B T$}

Taking $u^{\prime}=F^{\prime} / G^{\prime}$ as another solution of Eqs. (1.3), at the same time, $v^{\prime}=\ln \left(F^{\prime} / G^{\prime}\right), w^{\prime}=\ln \left(F^{\prime} G^{\prime}\right)$ also satisfy Eqs. (2.6). Let us consider

$$
\begin{aligned}
E_{1} & \equiv\left[i \mathscr{Y}_{t}\left(v^{\prime}\right)+P_{1}(t) \mathscr{Y}_{2 x}\left(v^{\prime}, w^{\prime}\right)+P_{2}(t) \mathscr{Y}_{2 y}\left(v^{\prime}, w^{\prime}\right)\right] \\
& -\left[i \mathscr{Y}_{t}(v)+P_{1}(t) \mathscr{Y}_{2 x}(v, w)+P_{2}(t) \mathscr{Y}_{2 y}(v, w)\right]=0,
\end{aligned}
$$

by using of the mixed variables:

$$
\begin{gathered}
v_{1}=\ln G^{\prime} / G, v_{2}=\ln F^{\prime} / F, v_{3}=\ln F^{\prime} / G, v_{4}=\ln G^{\prime} / F, \\
w_{1}=\ln G^{\prime} G, w_{2}=\ln F^{\prime} F, w_{3}=\ln F^{\prime} G, w_{4}=\ln G^{\prime} F,
\end{gathered}
$$

we can rewrite Eq. (3.1) as

$$
\begin{aligned}
E_{1} \equiv- & {\left[i \mathscr{Y}_{t}\left(v_{1}\right)-P_{1}(t) \mathscr{Y}_{2 x}\left(v_{1}, w_{1}\right)-P_{2}(t) \mathscr{Y}_{2 y}\left(v_{1}, w_{1}\right)\right] } \\
& +\left[i \mathscr{Y}_{t}\left(v_{2}\right)-P_{1}(t) \mathscr{Y}_{2 x}\left(v_{2}, w_{2}\right)-P_{2}(t) \mathscr{Y}_{2 y}\left(v_{2}, w_{2}\right)\right]+R_{1}=0,
\end{aligned}
$$

with the reminder term

$$
\begin{aligned}
R_{1} & =2 P_{1}(t)\left[v_{3,2 x}+v_{3, x}\left(v_{2}-v_{1}\right)_{x}\right]+2 P_{2}(t)\left[v_{3,2 y}+v_{3, y}\left(v_{2}-v_{1}\right)_{y}\right] \\
& =2 P_{1}(t) v_{3, x}\left[\ln v_{3, x}+\left(v_{2}-v_{1}\right)\right]_{x}+2 P_{2}(t) v_{3, y}\left[\ln v_{3, y}+\left(v_{2}-v_{1}\right)\right]_{y} .
\end{aligned}
$$

Therefore, Eq. (3.2) can be decoupled into

$$
\begin{array}{r}
i \mathscr{Y}_{t}\left(v_{1}\right)-P_{1}(t) \mathscr{Y}_{2 x}\left(v_{1}, w_{1}\right)-P_{2}(t) \mathscr{Y}_{2 y}\left(v_{1}, w_{1}\right)=\lambda(t), \\
i \mathscr{Y}_{t}\left(v_{2}\right)-P_{1}(t) \mathscr{Y}_{2 x}\left(v_{2}, w_{2}\right)-P_{2}(t) \mathscr{Y}_{2 y}\left(v_{2}, w_{2}\right)=\lambda(t), \\
\mathscr{Y}_{x}\left(v_{3}\right)-\mu_{1} e^{v_{1}-v_{2}}=0, \\
\mathscr{Y}_{y}\left(v_{3}\right)-\mu_{2} e^{v_{1}-v_{2}}=0,
\end{array}
$$


the BT of Eqs. (1.3) is obtained as

$$
\begin{array}{r}
{\left[i D_{t}-P_{1}(t) D_{x}^{2}-P_{2}(t) D_{y}^{2}\right] G^{\prime} \cdot G=\lambda(t) G^{\prime} G,} \\
{\left[i D_{t}-P_{1}(t) D_{x}^{2}-P_{2}(t) D_{y}^{2}\right] F^{\prime} \cdot F=\lambda(t) F^{\prime} F,} \\
D_{x} F^{\prime} \cdot G=\mu_{1} F G^{\prime}, \\
D_{y} F^{\prime} \cdot G=\mu_{2} F G^{\prime},
\end{array}
$$

where $\lambda(t)$ is an arbitrary function of variable $t, \mu_{1}$ and $\mu_{2}$ are constants.

Through Eqs. (3.4), we can get another solution from the known solution. For example, setting $F=G=1$ and substituting them into Eqs. (20), we obtain

$$
F^{\prime}=\lambda_{0}+\lambda_{1} e^{a x+b y+c(t)}, \quad G^{\prime}=\lambda_{2} e^{a x+b y+c(t)},
$$

where $a=\lambda_{2} /\left(\lambda_{1} \mu_{1}\right), b=\lambda_{2} /\left(\lambda_{1} \mu_{2}\right), c(t)=-i \int\left[a^{2} P_{1}(t)+b^{2} P_{2}(t)\right] d t, \lambda_{0}, \lambda_{1}$ and $\lambda_{2}$ are constants, the solution for Eqs. (1.3) is derived

$$
u^{\prime}=\frac{\lambda_{0}+\lambda_{1} e^{a x+b y+c(t)}}{\lambda_{2} e^{a x+b y+c(t)}}
$$

\subsection{Lax pair}

Through the expression

$$
\mathscr{Y}_{n z_{1}, m z_{2}}(w=\ln \psi, v=Q+w)=\psi^{-1} \sum_{i=0}^{n} \sum_{j=0}^{m} C_{n}^{i} C_{m}^{j} \mathscr{Y}_{i z_{1}, j z_{2}}(0, Q) \partial_{z_{1}}^{n-i} \partial_{z_{2}}^{m-j}
$$

where $\psi$ and $Q$ are both the functions of $z_{1}$ and $z_{2}$, Expression (2.3) is related to the Lax pair by use of the Hopf-Cole transformation [20,21].

Utilizing the Transformation (3.7), $v_{i}=\ln \varphi_{i}(i=1,2,3,4)$ and eliminating $v_{3}$ through $v_{3}=$ $v_{2}+v$, we get the bilinear system for Eqs. (2.7)

$$
\begin{aligned}
i \varphi_{1, t} & =P_{1}(t)\left[\varphi_{1, x x}+(w-v)_{x x} \varphi_{1}\right]+P_{2}(t)\left[\varphi_{1, y y}+(w-v)_{y y} \varphi_{1}\right], \\
i \varphi_{2, t} & =P_{1}(t)\left[\varphi_{2, x x}+(w-v)_{x x} \varphi_{2}\right]+P_{2}(t)\left[\varphi_{2, y y}+(w-v)_{y y} \varphi_{2}\right], \\
\varphi_{1, x} & =\frac{1}{\mu_{1}}\left(\varphi_{2, x x}+v_{x x} \varphi_{2}+\mu_{1} v_{x} \varphi_{1}-v_{x}^{2} \varphi_{2}\right), \\
\varphi_{2, y} & =\mu_{1} \varphi_{1}-v_{x} \varphi_{2}
\end{aligned}
$$

From the compatibility conditions $\varphi_{1, x t}=\varphi_{1, t x}$ and $\varphi_{2, y t}=\varphi_{2, t y}$, Eqs. (3.8) are equivalent to Eqs. (1.3), namely, Eqs. (3.8) are the Lax pair for Eqs.(1.3).

\section{Solutions for Eqs. (1.3)}

In this section, by using of the Hirota method [17] and symbolic computation [9, 34], we construct two special solutions for Eqs. (1.3): solitons and localized excitations. 
H.-P. Zhou et al.

\subsection{Solitons}

We expand $F$ and $G$ with respect to a small parameter $\varepsilon$ as follows:

$$
F=\varepsilon f_{1}+\varepsilon^{3} f_{3}+\cdots, \quad G=1+\varepsilon^{2} g_{2}+\varepsilon^{4} g_{4}+\cdots .
$$

Next, substituting Eqs. (4.1) into Eqs. (2.7) and collecting the coefficients of each order of $\varepsilon$, we can derive

$$
\begin{array}{ll}
\varepsilon^{0}: & \left(R D_{x}^{2}-D_{y}^{2}\right) 1 \cdot 1=0, \\
\varepsilon^{1}: & {\left[i D_{t}+P_{1}(t) D_{x}^{2}+P_{2}(t) D_{y}^{2}\right] f_{1} \cdot 1=0,} \\
\varepsilon^{2}: & \left(R D_{x}^{2}-D_{y}^{2}\right)\left(1 \cdot g_{2}+g_{2} \cdot 1\right)-K f_{1} f_{1}^{*}=0, \\
\varepsilon^{3}: & {\left[i D_{t}+P_{1}(t) D_{x}^{2}+P_{2}(t) D_{y}^{2}\right]\left(f_{1} \cdot g_{2}+f_{3} \cdot 1\right)=0 .}
\end{array}
$$

When truncating $F$ and $G$ as $F=\varepsilon f_{1}$ and $G=1+\varepsilon^{2} g_{2}$, and substituting them into Eqs. (4.2) we get $f_{1}=e^{\theta_{1}}, g_{2}=\delta_{1} e^{\theta_{1}+\theta_{1}^{*}}$. Without loss of generality, by setting $\varepsilon=1$, one-soliton solution is obtained as

$$
u=\frac{\varepsilon e^{\theta_{1}}}{1+\delta_{1} \varepsilon^{2} e^{\theta_{1}+\theta_{1}^{*}}}=\exp \left[i \theta_{1 I}-\frac{\ln \delta_{1}}{2}\right] \operatorname{sech}\left(\theta_{1 R}+\frac{\ln \delta_{1}}{2}\right)
$$

where

$$
\begin{aligned}
& \theta_{1}=a_{1} x+b_{1} y+c_{1}(t)+k_{1}, \\
& c_{1}(t)=C_{1}+i \int\left[a_{1}^{2} P_{1}(t)+b_{1}^{2} P_{2}(t)\right] d t, \\
& \delta_{1}=\frac{K}{2\left[R\left(a_{1}+a_{1}^{*}\right)^{2}-\left(b_{1}+b_{1}^{*}\right)^{2}\right]},
\end{aligned}
$$

$\theta_{1 R}$ and $\theta_{1 I}$ are respectively the real and imaginary components of $\theta_{1}$.

To constructe the two-soliton solution, we assume $F=\varepsilon f_{1}+\varepsilon^{3} f_{3}$ and $G=1+\varepsilon^{2} g_{2}+\varepsilon^{4} g_{4}$ with $f_{1}=e^{\theta_{1}}+e^{\theta_{2}}$, similarly, the two-soliton solution is obtained as

$$
u=\frac{f_{1}+f_{3}}{1+g_{2}+g_{4}}
$$

where

$$
\begin{aligned}
& \theta_{j}=a_{j} x+b_{j} y+c_{j}(t)+k_{j}, \quad(j=1,2), \\
& \left.c_{j}(t)=C_{j}+i \int\left[a_{j}^{2} P_{1}(t)+b_{j}^{2} P_{2}(t)\right]\right] d t, \quad(j=1,2), \\
& g_{2}=\delta_{11} e^{\theta_{1}+\theta_{1}^{*}}+\delta_{12} e^{\theta_{1}+\theta_{2}^{*}}+\delta_{21} e^{\theta_{2}+\theta_{1}^{*}}+\delta_{22} e^{\theta_{2}+\theta_{2}^{*}}, \\
& f_{3}=\delta_{121} e^{\theta_{1}+\theta_{2}+\theta_{1}^{*}}+\delta_{122} e^{\theta_{1}+\theta_{2}+\theta_{2}^{*}}, \\
& g_{4}=\delta_{1212} e^{\theta_{1}+\theta_{2}+\theta_{1}^{*}+\theta_{2}^{*}},
\end{aligned}
$$


with

$$
\begin{aligned}
\delta_{11}= & \frac{K}{2\left[R\left(a_{1}+a_{1}^{*}\right)^{2}-\left(b_{1}+b_{1}^{*}\right)^{2}\right]}, \quad \delta_{12}=\frac{K}{2\left[R\left(a_{1}+a_{2}^{*}\right)^{2}-\left(b_{1}+b_{2}^{*}\right)^{2}\right]}, \\
\delta_{21}= & \frac{K}{2\left[R\left(a_{2}+a_{1}^{*}\right)^{2}-\left(b_{2}+b_{1}^{*}\right)^{2}\right]}, \quad \delta_{22}=\frac{K}{2\left[R\left(a_{2}+a_{2}^{*}\right)^{2}-\left(b_{2}+b_{2}^{*}\right)^{2}\right]}, \\
\delta_{121}= & -\frac{P_{1}\left(a_{1}-a_{2}\right)\left[\delta_{11}\left(a_{1}+a_{1}^{*}\right)-\delta_{21}\left(a_{2}+a_{1}^{*}\right)\right]+P_{2}\left(b_{1}-b_{2}\right)\left[\delta_{11}\left(b_{1}+b_{1}^{*}\right)-\delta_{21}\left(b_{2}+b_{1}^{*}\right)\right]}{P_{1}\left(a_{1}+a_{2}^{*}\right)\left(a_{2}+a_{2}^{*}\right)+P_{2}\left(b_{1}+b_{2}^{*}\right)\left(b_{2}+b_{2}^{*}\right)}, \\
\delta_{122}= & -\frac{P_{1}\left(a_{1}-a_{2}\right)\left[\delta_{12}\left(a_{1}+a_{2}^{*}\right)-\delta_{22}\left(a_{2}+a_{2}^{*}\right)\right]+P_{2}\left(b_{1}-b_{2}\right)\left[\delta_{12}\left(b_{1}+b_{2}^{*}\right)-\delta_{22}\left(b_{2}+b_{2}^{*}\right)\right]}{P_{1}\left(a_{1}+a_{2}^{*}\right)\left(a_{2}+a_{1}^{*}\right)+P_{2}\left(b_{1}+b_{2}^{*}\right)\left(b_{2}+b_{1}^{*}\right)}, \\
\delta_{1212}= & -\frac{K\left(\delta_{121}+\delta_{121}^{*}+\delta_{122}+\delta_{12}^{*}\right)}{2 R\left(a_{1}+a_{1}^{*}+a_{2}+a_{2}^{*}\right)^{2}+2\left(b_{1}+b_{1}^{*}+b_{2}+b_{2}^{*}\right)^{2}} \\
& -\frac{\delta_{11} \delta_{22}\left[R\left(a_{1}+a_{1}^{*}-a_{2}-a_{2}^{*}\right)^{2}-\left(b_{1}+b_{1}^{*}-b_{2}-b_{2}^{*}\right)^{2}\right]}{R\left(a_{1}+a_{1}^{*}+a_{2}+a_{2}^{*}\right)^{2}+\left(b_{1}+b_{1}^{*}+b_{2}+b_{2}^{*}\right)^{2}} \\
& -\frac{\delta_{12} \delta_{21}\left[R\left(a_{1}+a_{2}^{*}-a_{2}-a_{1}^{*}\right)^{2}-\left(b_{1}+b_{2}^{*}-b_{2}-b_{1}^{*}\right)^{2}\right]}{R\left(a_{1}+a_{1}^{*}+a_{2}+a_{2}^{*}\right)^{2}+\left(b_{1}+b_{1}^{*}+b_{2}+b_{2}^{*}\right)^{2}},
\end{aligned}
$$

where $P_{1}=P_{1}(t)$ and $P_{2}=P_{2}(t)$.

Based on the previous solutions' expressions, we show the propagation and collisions of solitons in graphics.

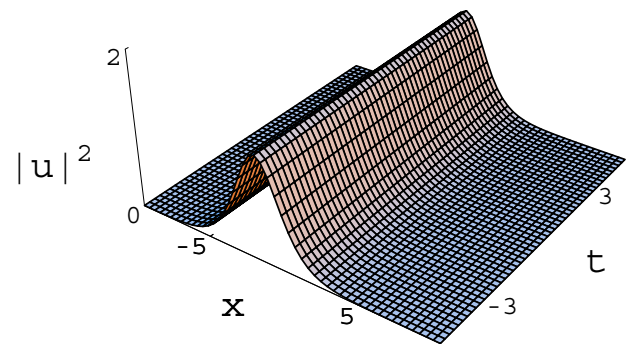

(a)

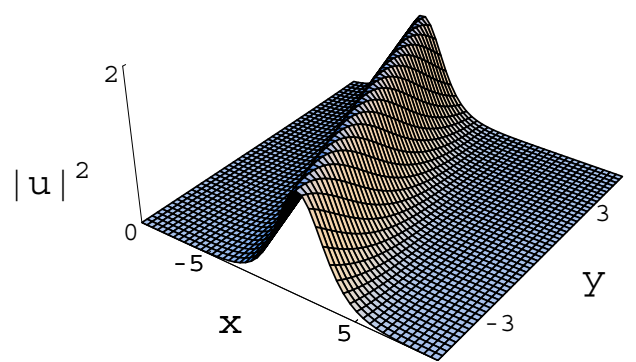

(b)

Figs. 1 One soliton via Expressions (4.3). Parameters are $k_{1}=0.1, a_{1}=-0.5, b_{1}=-0.3, R=3, K=1, P_{1}(t)=2 \sin (t)$ and $P_{2}(t)=\sin (t)$. Respectively, (a) shows the soliton at $y=0$ and (b) is $t=0$.

As shown in Figs. 1, though the soliton's velocity ( $x$ axial velocity $v_{1}=-\frac{a c(t)}{a^{2}+b^{2}}$ and $y$ axial velocity $\left.v_{2}=-\frac{b c(t)}{a^{2}+b^{2}}\right)$ changes with the time $t$, variable coefficients have no effect on the profile of the soliton. 


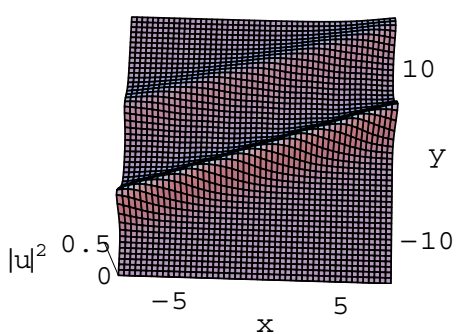

$(\mathrm{a}: t=-4)$

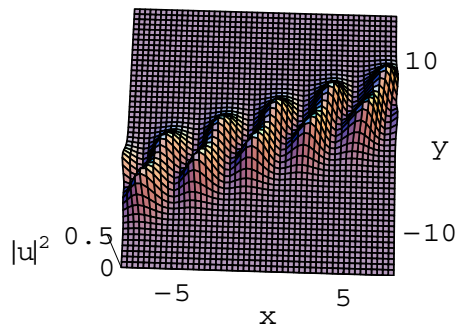

$(\mathrm{b}: t=-1)$

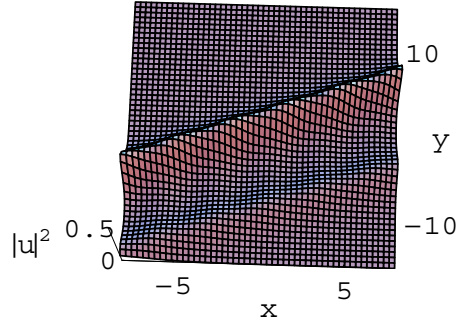

$(\mathrm{c}: t=2)$

Figs. 2 Interaction of two parallel bell-shape solitons via Expressions (4.4) with constant coefficients at different times. Parameters are $k_{1}=0, k_{2}=-2, a_{1}=0.4, a_{2}=0.31+2 i, b_{1}=-0.6, b_{2}=-0.49, R=3, K=1, P_{1}(t)=1$ and $P_{2}(t)=4$.

Figs. 2 describe the collision of the two parallel solitons. At $t=-4$, the shorter soliton is about to interact with the taller one, at $t=-1$ they are overlapping, and at $t=2$ they recover their original shapes and respectively move ahead as the beginning. We can see that the solitons collide elastically, which can also be shown in Figs. 3.

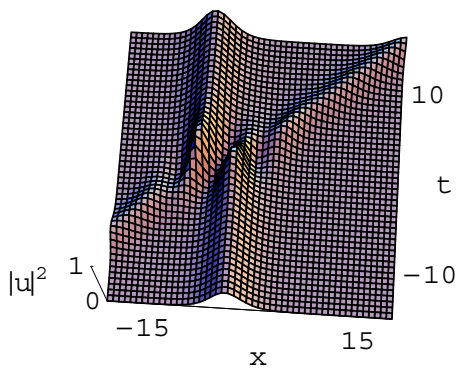

(a: $y=-5)$

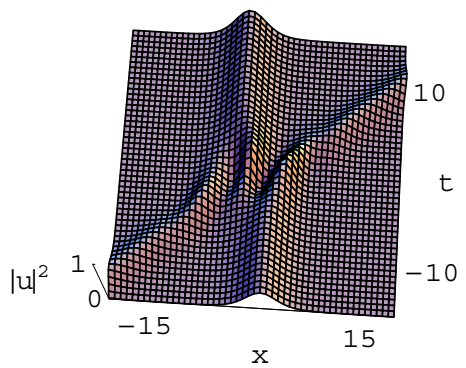

(b: $y=0)$

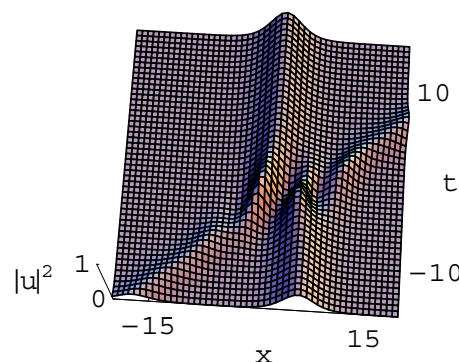

(c: $y=5)$

Figs. 3 Interaction of two solitons via Expressions (4.4) with constant coefficients. Parameters are $k_{1}=1.2, k_{2}=0$, $a_{1}=0.33, a_{2}=0.34+0.8 i, b_{1}=-0.3, b_{2}=-0.5, R=3, K=1, P_{1}(t)=1$ and $P_{2}(t)=4$.

As seen in Figs. 3, two obliquely moving solitons display the elastic interaction and keep their original shapes and velocities invariant after the interaction with the phase shift in the $x-t$ plane. 


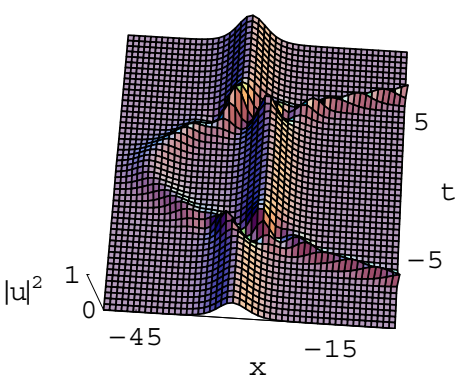

(a: $y=-30)$

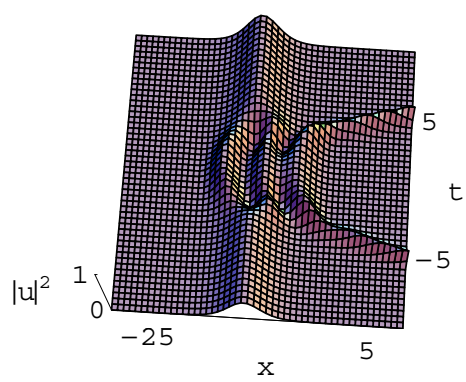

(b: $y=-10)$

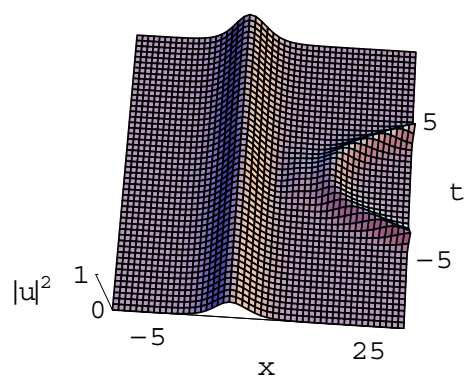

$(\mathrm{c}: y=10)$

Figs. 4 Interaction of two solitons via Expressions (4.4) with variable coefficients. Parameters are $k_{1}=1.2, k_{2}=0$, $a_{1}=0.33, a_{2}=0.34+0.8 i, b_{1}=-0.3, b_{2}=-0.5, R=3, K=1, P_{1}(t)=t$ and $P_{2}(t)=4 t$.

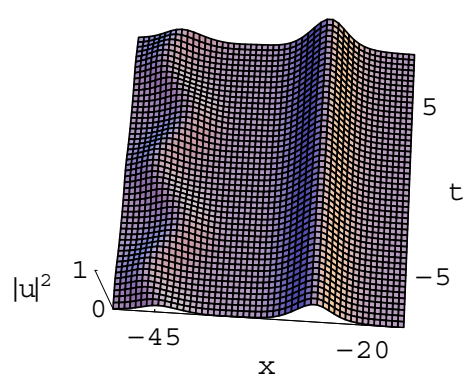

(a: $y=-30)$

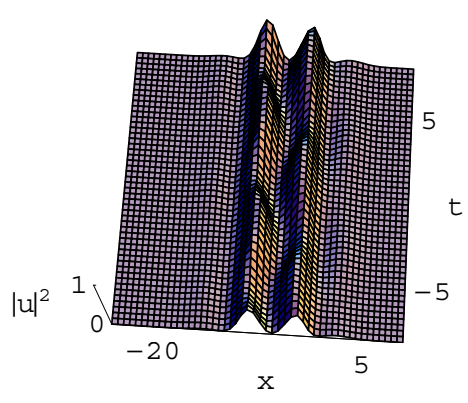

(b: $y=-5$ )

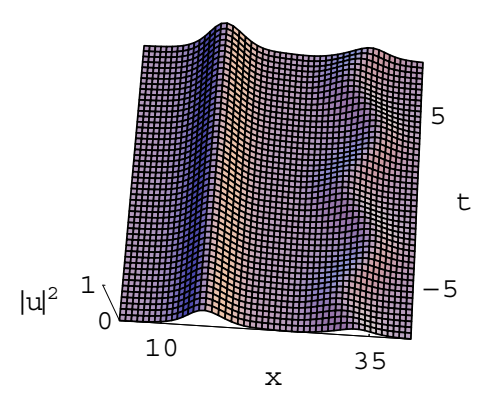

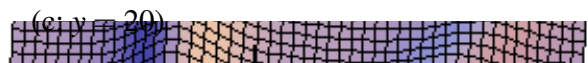

Figs. 5 Interaction of two solitons via Expressions (4.4) with variable coefficients. Parameters are $k_{1}=1.2, k_{2}=0$, $a_{1}=0.33, a_{2}=0.34+0.8 i, b_{1}=-0.3, b_{2}=-0.5, R=3, K=1, P_{1}(t)=\sin (t)$ and $P_{2}(t)=4 \sin (t)$.

From Figs. 3-5, we observe the different effects on solitons between the constant coefficients and variable coefficients where all the parameters are same besides $P_{1}(t)$ and $P_{2}(t)$. Figs. 3 show the two unparallel solitons' collision at different $y$ with constant coefficients $P_{1}(t)=1$ and $P_{2}(t)=4$. For Figs. 4 and 5, solitons no longer keep the rectilinear propagation and display different shapes because of the effect of variable coefficients. In Figs. 4, one of the soliton is parabolically typed with coefficients $P_{1}(t)=t$ and $P_{2}(t)=4 t$. At $y=-30$ two solitons intersect at the two parabolic branches, at $y=-10$ they intersect at the parabolic vertex, and at $y=10$ they separate and move ahead respectively. In Figs. 5, we can observe that one of the solitons possesses the periodic characteristic with $P_{1}(t)=\sin (t)$ and $P_{2}(t)=4 \sin (t)$. In addition, we can modulate the coefficients to obtain more soliton structures.

\subsection{Localized excitations}

The dromions, which are the exponentially localized soliton solutions in higher dimensions, have attracted attention $[3,11,16,25,27]$. Dromions may either decay due to the dispersion to be enhanced by the static field or exhibit blowup due to nonlinearity in a finite time $[3,30]$. The multidromion solutions were constructed by use of the BT [4], later solved by the IST [1, 4] and 
Hirota method [15]. Recent years, the variable separation method has been revisited and improved for the (2+1)-dimensional equations to obtain the local solutions.

With the transformation $x=\frac{1}{\sqrt{R}} x^{\prime}+y^{\prime}, y=\frac{1}{\sqrt{R}} x^{\prime}-y^{\prime}, t=t^{\prime}$ and $P_{1}(t)=R P_{2}(t)$, we can reduce equations Eqs. (2.7) as

$$
\begin{aligned}
& {\left[i D_{t^{\prime}}+2 P_{1}(t) D_{x^{\prime}}^{2}+2 P_{2}(t) D_{y^{\prime}}^{2}\right] F \cdot G=0,} \\
& D_{x^{\prime}} D_{y^{\prime}} G \cdot G-\frac{K}{4} F F^{*}=0 .
\end{aligned}
$$

Through the variable separation [25, 35], assuming $u=F / G, \phi=2 C(\ln G)_{x^{\prime} x^{\prime}}+p_{0}+q_{0}$, $G=\rho_{0}+\rho_{1} p+\rho_{2} q+\rho_{3} p q$ and $F=p_{1} q_{1} e^{i r+i s}$, substituting them into Eqs. (4.5), the solution of Eqs. (1.3) is obtained

$$
u=\frac{2 o_{1} o_{2} \sqrt{\left(\rho_{0} \rho_{3}-\rho_{1} \rho_{2}\right) p_{x^{\prime}} q_{y^{\prime}}} e^{i r+i s}}{\rho_{0}+\rho_{1} p+\rho_{2} q+\rho_{3} p q},
$$

where $p_{0}=p_{0}\left(x^{\prime}, t^{\prime}\right), q_{0}=q_{0}\left(y^{\prime}, t^{\prime}\right), p=p\left(x^{\prime}, t^{\prime}\right), q=q\left(y^{\prime}, t^{\prime}\right), r=\left(x^{\prime}, t^{\prime}\right), s=s\left(y^{\prime}, t^{\prime}\right), p_{1}=p_{1}\left(x^{\prime}, t^{\prime}\right)$, $q_{1}=q_{1}\left(y^{\prime}, t^{\prime}\right)$ and $o_{1}^{2}=o_{2}^{2}=1$. The intensity of the wave envelope is given by

$$
|u|^{2}=\frac{4\left(\rho_{0} \rho_{3}-\rho_{1} \rho_{2}\right) p_{x^{\prime}} q_{y^{\prime}}}{\left(\rho_{0}+\rho_{1} \rho_{2}+\rho_{2} q+\rho_{3} p q\right)^{2}} .
$$

When $p$ and $q$ satisfy the relation $\left(\rho_{0} \rho_{3}-\rho_{1} \rho_{2}\right) p_{x^{\prime}} q_{y^{\prime}}>0, u$ is a coherent soliton solution localized in some directions or in all directions. Choosing different $p\left(x^{\prime}, t^{\prime}\right)$ and $q\left(y^{\prime}, t^{\prime}\right)$, different excitation patterns such as the dromion patterns, solitoff patterns, resonant solitoff patterns will arise.

For instance, selecting $p$ and $q$ as

$$
\begin{aligned}
p\left(x^{\prime}, t^{\prime}\right) & =m_{j} \sum_{j=1}^{M} \exp \left[a_{j} x^{\prime}+c_{j} t^{\prime}+k_{j}\right], \\
q\left(y^{\prime}, t^{\prime}\right) & =n_{j} \sum_{j=1}^{N} \exp \left[b_{j} y^{\prime}+d_{j} t^{\prime}+l_{j}\right],
\end{aligned}
$$

where $m_{j}, n_{j}, a_{j}, b_{j}, c_{j}, d_{j}, k_{j}$ and $l_{j}$ are arbitrary constants and $M, N$ are the positive integers, we get the dromion solutions.

\section{Conclusions}

We have investigated Eqs. (1.3) which have the applications in the ultra-relativistic degenerate dense plasmas and Bose-Einstein condensates. By virtue of the Bell polynomials and symbolic computation, Bilinears (2.7), BT (3.4) and Lax pair (3.8) have been obtained. Based on the Hirota method, we have obtained the soliton solutions in Expressions (4.3) and (4.4), and the propagation characteristics and elastic collisions of the solitons have also been discussed. From Figs. 2 and 3, we have shown that two-soliton with constant coefficients have the elastic collision, while for the variable coefficients in Figs. 4 and 5, solitons no longer keep the rectilinear propagation and display the different shapes. In addition, by means of the variable separation, localized excitations have been expressed in Eq. (4.6), which is a coherent soliton solution localized in some directions or in all directions. 


\section{Acknowledgments}

We would like to express our gratitude to the referees for their valuable comments and suggestions. This work has been supported by the National Natural Science Foundation of China under Grant No. 60772023, No. 11161042 and No. 11272065, by the Fundamental Research Funds for the Central Universities of China under Grant No. 2011BUPTYB02, and by the Specialized Research Fund for the Doctoral Program of Higher Education (No. 200800130006), Chinese Ministry of Education.

\section{References}

[1] ARKADIEV V A, POGREBKOV A K and POLIVANOV M C, Inverse scattering transform method and soliton solutions for the Davey-Stewartson II equation, Phys D 36 (1989), 189.

[2] BELL E T, Exponential polynomials, Ann. Math 35 (1934), 258.

[3] BESSE C, MAUSER N J and STIMMING H P, Numerical study of the Davey-Stewartson system, ESAIM: Math. Model. Numer. Anal. 38 (2004), 1035.

[4] BOITI M, LEON J P, MARTINA L and PEMPINELLI F, Scattering of localized solitons in the plane, Phys. Lett. A 132 (1988), 432.

[5] DAVEY A and STEWARTSON K, On three-dimensional packets of surfaces waves, Proc. Roy. Soc. London Ser. A 338 (1974), 101.

[6] FOKAS A S and SANTINI P M, Coherent structures in multidimensions, Phys. Rev. Lett. 63 (1989), 1329.

[7] FREEMAN N C, Soliton solutions of non-linear evolution equations, J. Appl. Math. 32 (1984), 125.

[8] GANESANT S and LAKSHMANANI M, Singularity-structure analysis and Hirota's bilinearisation of the Davey-Stewartson equation, J. Phys. A 20 (1987), 1143.

[9] GAO Y T and TIAN B, Cylindrical Kadomtsev-Petviashvili model, nebulons and symbolic computation for cosmic dust ion-acoustic waves, Phys. Lett. A 349 (2006), 314.

[10] GEN D P, PU F C and ZHAO B H, Exact solution for quantum Davey-Stewartson I system, Phys. Rev. Lett. 65 (1990), 3227.

[11] GHOSH S S, SEN A and LAKHINA G S, Dromion solutions for nonlinear electron acoustic waves in space plasmas, Nonlinear Processes Geophys. 9 (2002), 463.

[12] GILSON C, LAMBERT F, NIMMO J and WILLOX R, On the combinatorics of the Hirota D-operators, Proc. Roy. Soc. London Ser. A 452 (1996), 223.

[13] HASEGAWA A and TAPPERT F, Transmission of stationary nonlinear optical pulses in dispersive dielectric fibers. I. Anomalous dispersion, Appl. Phys. Lett 23 (1973), 142.

[14] HAUS H A and WONG W S, Solitons in optical communications, Rev. Mod. Phys. 68 (1996), 423.

[15] HIETARINTA J, One-dromion solutions for generic classes of equations, Phys. Lett. A 149 (1990), 113.

[16] HIETARINTA J and HIROTA R, Multidromion solutions to the Davey-Stewartson equation, Phys. Lett. A 145 (1990), 237.

[17] HIROTA R, The direct method in soliton theory, (Springer, Berlin, 1980).

[18] HUANG G X, DENG L and HANG C, Davey-Stewartson description of two-dimensional nonlinear excitations in Bose-Einstein condensates, Phys. Rev. E 72 (2005), 036621.

[19] JAFARI H and ALIPOUR M, Numerical Solution of the Davey-Stewartson Equations using Variational Iteration Method, World. Appl. Sci. J 8 (2010), 814. 
[20] LAMBERT $\mathrm{F}$ and SPRINGAEL J, On a direct procedure for the disclosure of Lax pairs and Bäklund transformations, Chaos Solitons Fractals 12 (2001), 2821.

[21] LAMBERT F and SPRINGAEL J, Soliton equations and simple combinatorics, Acta. Appl. Math. 102 (2008), 147.

[22] LEO R A, MANCARELLA G, SOLIANI G and SOLOMBRINO L, On the Painlevé property of nonlinear field equations in 2+1 dimensions: The Davey-Stewartson system, J. Math. Phys. 29 (1988), 2666.

[23] LEVIA D, PILLONIA L and SANTINI P M, Bäcklund transformations for nonlinearevolutionequations in 2+1 dimensions, Phys. Lett. A 81 (1981), 419.

[24] LI Y, Bäcklund-Darboux transformations and melnikov analysis for Davey-Stewartson II equations, J. Nonlinear Sci. 10 (2000), 103.

[25] LOU S Y, Dromions, dromion lattice, breather and instantons of the DS equation, Phys. Scr 65 (2002), 7.

[26] MAFIAS M and SANTINI P M, Solutions of the Davey-Stewartson II equation with arbitrary rational localization and nontrivial interaction, Phys. Lett. A 227 (1997), 325.

[27] MATVEEV V B and SALLE M A, Darboux transformations and solitons (Springer-Verlag, Berlin, 1991).

[27] MCCONNELL M, FOKAS A S and Pelloni B, Localised coherent solutions of the DSI and DSII equations-a numerical study, Math. Compute. Simul. 69 (2005), 424.

[29] MISRA A P and SHUKLA P K, Stability and evolution of wave packets in strongly coupled degenerate plasmas, Phys. Rev. E 85 (2012), 026409.

[30] MISRA A P and SHUKLA P K, Modulational instability and nonlinear evolution of twodimensional electrostatic wave packets in ultra-relativistic degenerate dense plasmas, Phys. Plasmas 18 (2011), 042308.

[31] MISRA A P, MARKLUND M, BRODIN G and SHUKLA P K, Stability of two-dimensional ion-acoustic wave packets inquantum plasmas, Phys. Plasmas 18 (2011, 042102.

[32] SKUPIN S, BANG O, EDMUNDSON D and KROLIKOWSKI W, Stability of twodimensional spatial solitons in nonlocal nonlinear media, Phys. Rev. E 73 (2006), 066603.

[33] TANG X Y, LOU S Y and ZHANG Y, Localized excitations in (2+1)-dimensional systems, Phys. Rev. E 66 (2002), 046601.

[34] TIAN B and GAO Y T, Symbolic-computation study of the perturbed nonlinear Schrödinger model in inhomogeneous optical fibers, Phys. Lett. A 342 (2005), 228.

[35] WANG R J and HUANG Y C, Exact solutions and excitations for the Davey-Stewartson equations with nonlinear and gain terms, Eur. Phys. J. D 57 (2012), 395.

[36] WU Y and DENG L, Ultraslow optical solitons in a cold four-state medium, Phys. Rev. Lett. 93 (2004) 1439041. 